\title{
IDENTIFYING THE FINANCING PATTERN PROBLEMS OF DILAPIDATED URBAN HOUSING RENEWAL SYSTEM IN ZHEJIANG, CHINA: CASE STUDY OF JINSHOU PROJECT IN ZHOUSHAN
}

\author{
中国浙江の危房改造システムの資金面の問題点：舟山の「金寿新村」プロジェクトを対象に \\ Li GUAN ${ }^{* 1}$ and Takashi ARIGA*2 \\ 管 理, 有賀 隆
}

\begin{abstract}
A dualistic system of private urban housing renewal consisting of marketized "Old City Renewal" and government voluntary "Dilapidated Urban Housing Renewal" has been established in China since 2015. Focusing on the latter DUHR mode, this study aims to identify its problems from the perspective of financing pattern. Jinshou Project in Zhoushan is selected as a representative case to clarify the DUHR mode in Zhejiang Province. Through analyzing the statistics of project funding, this paper argues that the DUHR mode completely relies on public funding, and is hard to tackle the increasing number of dilapidated housing.
\end{abstract}

Keywords: Dilapidated Urban Housing Renewal, Old City Renovation, Financing pattern, China 危房改造, 旧城改造, 資金の流れ, 中国

\section{Introduction}

\subsection{Research background}

\section{Evolution of Chinese urban housing system}

The Chinese social welfare urban housing system established in the 1950s transformed into a market-oriented housing system through a step by step housing reform from 1978 to 1998. Large amount of commodity housing was intentionally constructed in Chinese cities after 1978, so were most former public housing purchased by employees of state-owned enterprises. By now an urban housing provision system dominated by private housing has been established in China. ${ }^{\left.{ }^{* 1}\right)}$ The issues discussed in this paper belong to the domain of private housing. ${ }^{\left.{ }^{2}\right)}$

\section{Dilapidated urban housing in China}

Due to a variety of reasons - low design standard (Lue et al., 2001; Qu, 2011), poor construction quality before the 1990s, and residents' improper usage (Suo, 2013) — an increasing number of buildings constructed during early 1980s to early 1990s are faced with aging of facilities, outdated layouts, and most importantly damage of building structure. ${ }^{* 3)}$

The term "Dilapidated Housing" infers that the building structure has been seriously damaged or the load-bearing component is in danger and may at any time lose the stability and load-bearing capacity. ${ }^{\left.{ }^{*}\right)}$ Recent years, frequent collapse accident ${ }^{{ }^{*} \text { ) }}$ in old city areas have caused wide public concern, urging the central government to take concrete measures.

\section{Dualistic system of private urban housing renewal in China}

Under the Chinese urban renewal framework before 2015, dilapidated housing areas on best sites would undergo Old City Renovation (OCR, “旧城改造”范) before long, which is a marketized process that the local government first requisitions properties from homeowners, and then sells the land to developers. Developers may maximize their benefits by building high-grade residences and approximating the FAR limit ${ }^{* 7)}$ restricted by urban planning.

While for other old residential areas located in the old city area without the optimal marketability, developers seldom show interest, and few substantial measures could be taken. Such residential areas in the inner city gradually turn into the leftovers in housing market, with their physical condition deteriorating. Yet these residential areas are still to a large extent occupied, due to
*1 Grad. School of Creative Science and Engineering, Waseda University, M.Arch.

*2 Prof., Grad. School of Creative Science and Engineering, Waseda University, Ph.D.
早稲田大学理工学術院創造理工学研究科 博士後期課程 早稲田大学理工学術院創造理工学研究科＼cjkstart教授·Ph.D. 
Table 1 Distinctions between Chinese OCR and DUHR system

\begin{tabular}{l|l|l|}
\hline & \multicolumn{1}{|c|}{$\begin{array}{c}\text { OCR (旧城改造) } \\
\text { - Old City Renovation }\end{array}$} & \multicolumn{1}{|c|}{$\begin{array}{c}\text { DUHR (危房改造) } \\
\text { - Dilapidated Urban Housing } \\
\text { Renewal }\end{array}$} \\
\hline Targeted areas & $\begin{array}{l}\text { Dilapidated areas with the best } \\
\text { marketability }\end{array}$ & $\begin{array}{l}\text { Areas excluded from OCR } \\
\text { projects }\end{array}$ \\
\hline $\begin{array}{l}\text { Initial goal } \\
\text { To optimize the urban function } \\
\text { in old city area through } \\
\text { redevelopment, and to renew its } \\
\text { physical environment }\end{array}$ & $\begin{array}{l}\text { To eliminate the life risks } \\
\text { caused by collapse of buildings }\end{array}$ \\
\hline $\begin{array}{l}\text { Stakeholders } \\
\text { involved }\end{array}$ & $\begin{array}{l}\text { Local government } \\
\text { Developer } \\
\text { Homeowner }\end{array}$ & $\begin{array}{l}\text { Local government } \\
\text { Homeowner }\end{array}$ \\
\hline $\begin{array}{l}\text { Profit for } \\
\text { government and } \\
\text { developer }\end{array}$ & Yes & No \\
$\begin{array}{l}\text { Rely on } \\
\text { government's re- } \\
\text { planning scheme }\end{array}$ & Yes & No \\
\hline $\begin{array}{l}\text { Rely on public } \\
\text { appropriation }\end{array}$ & No & Yes \\
\hline
\end{tabular}

the convenient transportation and amenities of the inner-city area, homeowner's limited economic condition, or the aging of residents.

In mid-2015, a new mode of private urban housing renewal is carried out by the State Council, under the name of Dilapidated Urban Housing Renovation (DUHR, “危房改造 "*8). Working as a complement to OCR, the DUHR mode is a government voluntary mode that focuses on housing renewal issues excluded from OCR areas. According to government's administrative files ${ }^{* 9)}$, "all Class D Dilapidated Housing ${ }^{*}$ in metropolitan areas should be evacuated by the end of 2017 ", which means a total number of 18 million housing units will be involved from 2015 to 2017 across the nation. With local governments obeying this schedule and competing to accomplish the administrative assignment, a government-dominated movement of renewing urban dilapidated housing takes place.

In this way, a dualistic system of private housing renewal is established in China (Table 1). On one hand, there is the fully marketized OCR approach led by local government and property developers, which squeezes land value of the best sites; on the other hand, there is the government dominated voluntary DUHR mode, which aims to eliminate the life risks caused by collapse of buildings excluded from OCR with the help of public funding. Through examining a typical case, this study focuses on the latter DUHR mode, which has not drawn so much attention as the OCR mode in academic research.

Another related policy named Dilapidated Rural Housing Renovation (“農村危房改造”), which deals with dilapidated housing on rural land by granting monetary subsidy, will not be discussed in this paper, due to the different land system and housing system of urban and rural area in China ${ }^{\left.{ }^{*} 10\right)}$.

\subsection{Research purpose}

There is no doubt that DUHR movement could eliminate currently diagnosed dilapidated buildings in the short term. Nonetheless, such movement guided by radical administrative subcontract is also correlated to a variety of social, economic, and physical environment issues.

The purpose of this paper is to identify the problems of DUHR system in Zhejiang Province in terms of financing pattern with original findings. This paper argues that, the DUHR mode completely relies on public funding, and is hard to tackle the increasing number of dilapidated housing in the future.

\subsection{Research method}

This paper uses case study method to identify the problems of DUHR system. Based on a background study, a representative case is selected in order to clarify the DUHR mode.

Main statistics of project funding, which are collected from Subdistrict Office and Bureau for Housing and Urban-rural Development (BHUD), is used to reveal the financing pattern of case study. Besides, the impact on physical environment is collected by field survey in old residential area and new resettlement residential areas.

\subsection{Former works}

Research on the government voluntary DUHR mode is still scarce in China, due to the fact that DUHR policy is relatively new, and accordingly the first DUHR projects were just completed in 2016. Among related studies, the OCR mode has been under research ever since the nation-wide urban renewal and housing reform took place in the early 1990s. Related studies have been dedicated to the interest balance of different stakeholders in OCR projects.

Lue (1997) observed that the growing real estate industry undermined OCR's initial intention to maintain original residents on site while redeveloping the old city. It also has been widely acknowledged that local government and developers profit themselves from the emerging real estate industry, and it is local elites that have benefited more than local residents (Fang and Zhang, 2003; Luo and Shen, 2006; Hu and Zhang, 2015). Shih (2010) argues that Chinese laws have promoted the booming of real estate industry and laid foundation for disputed relocation. It could be summarized that local residents with limited power and privileges could not get a profit in proportion to local government and developers from urban renewal.

Focusing on the new DUHR mode, this paper attempts to identify its financing problems through an in-depth case study.

\section{Jinshou Project case study as a representative of DUHR}

The project of Building No.4 of Jinshou Residential Area (金寿新村 4 幢) in Dinghai metropolitan area, Zhoushan ${ }^{\text {*11) }}$ (Fig. 1) is selected as a typical case of DUHR in Zhejiang. The representativeness of Jinshou case lies in the fact that:

1) Current condition of the residential area: Being one of Zhoushan's first commodity housing projects, Jinshou Residential Area is a typical gated community constructed at the beginning stage of housing reform in the 1980s (Fig. 2). Like most residential areas constructed in the same era, today it is suffering from deterioration of building structure: aside from Building No.4 which was diagnosed as Class D Dilapidated Housing, 22 other 

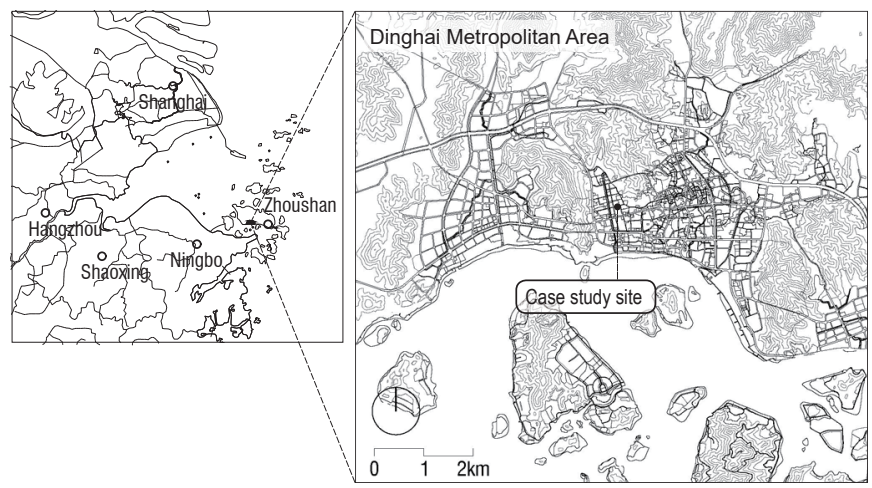

Fig. 1 Location of Dinghai metropolitan area and case study project

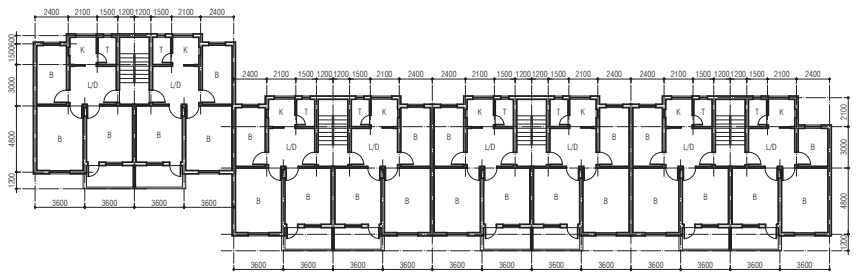

Fig. 3 Typical floor plan of Building No.4

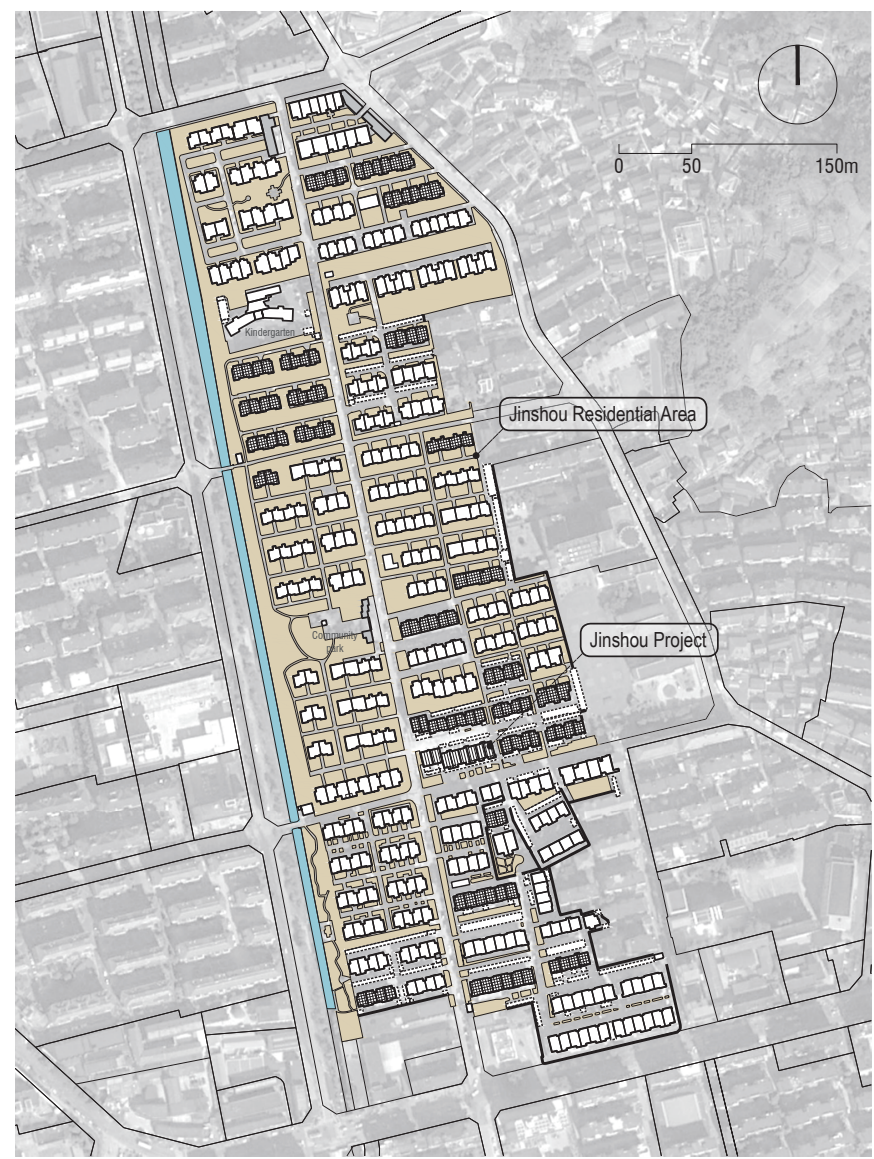

Class D dilapidated housing Shop

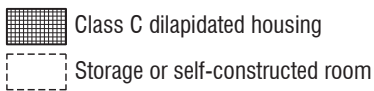

Storage or self-constructed room

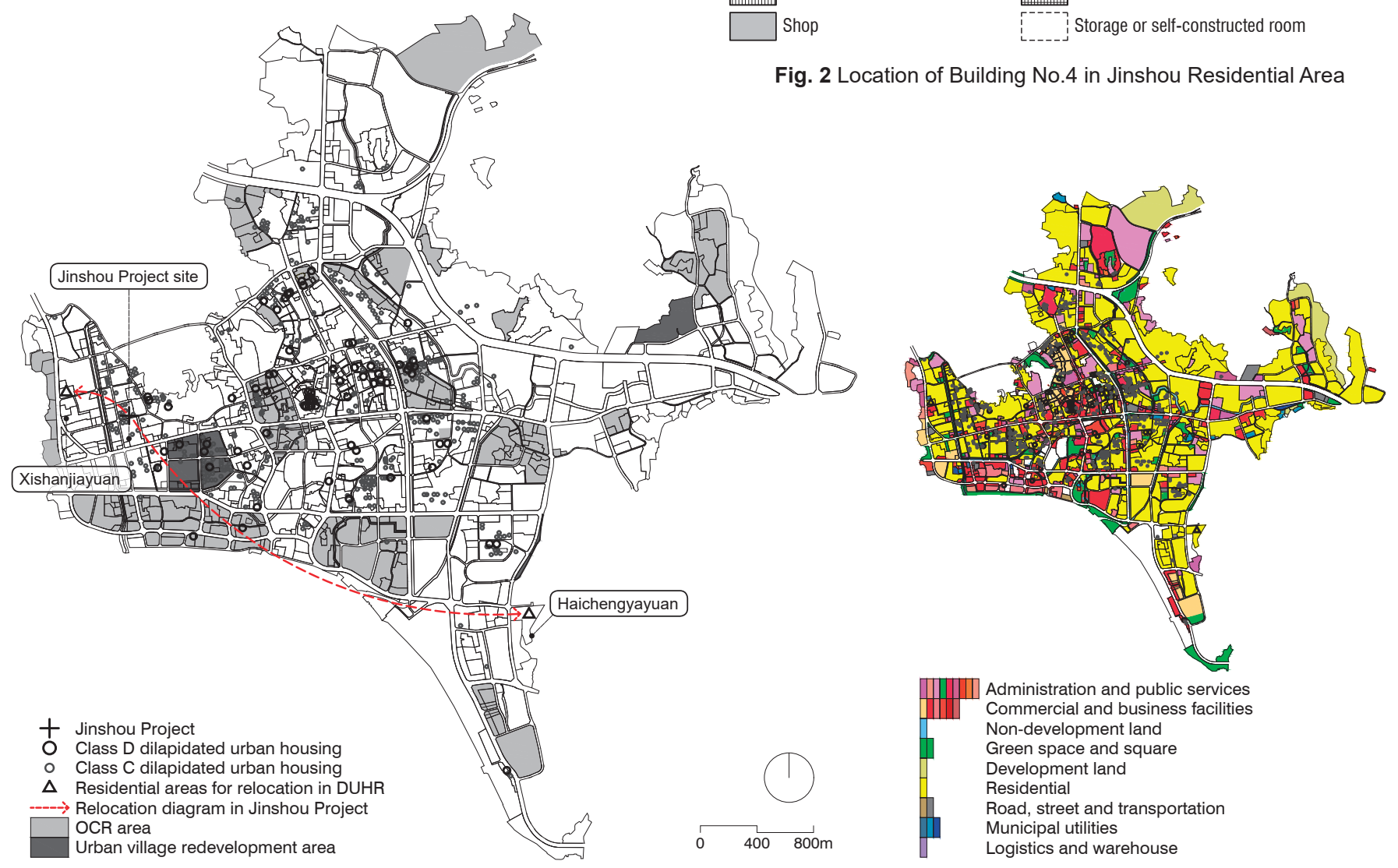

Fig. 4 Location of DUHR projects and relocation housing areas in Dinghai metropolitan area (left: overlapped with OCR areas; right: overlapped with land zoning) 
Table 2-1 DUHR strategies provided by government

\begin{tabular}{l|l}
\hline \multicolumn{1}{c|}{ Type } & \multicolumn{1}{c}{ Strategy } \\
\hline \multirow{2}{*}{ In-situ resettlement } & a) Structure reinforcement \\
\cline { 2 - 2 } Relocation & b) In-situ reconstruction \\
\hline \multirow{2}{*}{ c) Property swap (with local government) } \\
\hline
\end{tabular}

Table 2-2 Strategies adopted in four cities in Zhejiang Province

\begin{tabular}{|c|c|c|c|c|c|c|}
\hline \multirow{2}{*}{ City } & \multirow{2}{*}{ District } & \multirow{2}{*}{ Subdistrict } & \multicolumn{3}{|c|}{ Strategy adopted } & \multirow{2}{*}{ Total } \\
\hline & & & a) & b) & \begin{tabular}{l|l} 
c) & d) \\
\end{tabular} & \\
\hline \multirow{9}{*}{$\begin{array}{l}\text { Hangzhou } \\
(2017)\end{array}$} & \multirow{5}{*}{ Shangcheng } & Nanxing & 10 & - & 1 & 11 \\
\hline & & Qingbo & 2 & - & - & 2 \\
\hline & & Hubin & 3 & 1 & - & 4 \\
\hline & & \begin{tabular}{|l} 
Ziyang \\
\end{tabular} & 1 & - & 31 & 32 \\
\hline & & Subtotal & 16 & 1 & 32 & 49 \\
\hline & Xiacheng & Subtotal & 0 & 5 & 0 & 5 \\
\hline & Jianggan & Subtotal & 4 & 2 & 59 & 65 \\
\hline & Gongshu & Subtotal & 1 & 6 & 0 & 12 \\
\hline & Xihu & Subtotal & 2 & 0 & 0 & 2 \\
\hline \multirow{7}{*}{$\begin{array}{l}\text { Ningbo } \\
(2017)\end{array}$} & \multirow{7}{*}{ Jiangbei } & Kongpu & - & - & 6 & 6 \\
\hline & & Zhuangqiao & 2 & - & 5 & 7 \\
\hline & & Cicheng & 1 & - & 2 & 3 \\
\hline & & Wenjiao & - & - & 2 & 2 \\
\hline & & Hongtang & 1 & - & - & 1 \\
\hline & & Baisha & 1 & - & 3 & 4 \\
\hline & & \begin{tabular}{|l} 
Subtotal \\
\end{tabular} & 5 & 0 & 18 & 23 \\
\hline \multirow{6}{*}{$\begin{array}{l}\text { Shaoxing } \\
(2017)\end{array}$} & \multirow{6}{*}{ Yuecheng } & Beihai & 3 & - & 2 & 5 \\
\hline & & \begin{tabular}{|l|} 
Chengnan \\
\end{tabular} & - & - & 1 & 1 \\
\hline & & Tashan & 1 & - & & 1 \\
\hline & & \begin{tabular}{|l} 
Fushan \\
\end{tabular} & 10 & - & 1 & 11 \\
\hline & & \begin{tabular}{|l|} 
Jishan \\
\end{tabular} & 1 & - & 3 & 4 \\
\hline & & \begin{tabular}{|l|} 
Subtotal \\
\end{tabular} & 15 & 0 & 7 & 22 \\
\hline $\begin{array}{l}\text { Zhoushan } \\
\text { (2015-2017) }\end{array}$ & Dinghai & Subtotal & 27 & 0 & 48 & 75 \\
\hline
\end{tabular}

The locations of cities are marked on Fig.1.

Table 2-3 Strategeis adopted in 75 DHUR projects in Dinghai

\begin{tabular}{|c|c|c|c|c|c|c|c|c|c|c|c|}
\hline \multirow{2}{*}{ No. } & \multirow{2}{*}{ Location } & \multicolumn{4}{|c|}{ Strategy } & & \multirow{2}{*}{ Location } & \multicolumn{4}{|c|}{ Strategy } \\
\hline & & a) & & & & & & & & & d) \\
\hline 01 & Changdong No. 46 & & & $\circ$ & & 39 & Chaishui Aly. No. 14 & 0 & & & \\
\hline 02 & Dongguan No. 138 & & & $\circ$ & & 40 & Chaishui Aly. No. 35 & 0 & & & \\
\hline 03 & Dongguan No. 141 & & & $\circ$ & & 41 & Jiangjunqiaoxia No. 16 & $\circ$ & & & \\
\hline 04 & Wengshan No. 6 & & & $\circ$ & & 42 & Zuojiaochenggen No. 33 & 0 & & & \\
\hline 05 & Donggang No. 19-12 & & & 0 & & 43 & Sangyuan Aly. No. 39 & 0 & & & \\
\hline 06 & Xiyuan No. 29 & & & $\circ$ & & 44 & Sangyuan Aly. No. 10 & & & 0 & \\
\hline 07 & Qingleitou Rd. No. 89-3 & & & $\circ$ & & 45 & Jianguo Rd. No. 44 & & & 0 & \\
\hline 08 & Aaoshan No. 14 & & & $\circ$ & & 46 & Sangyuan Aly. No. 23 & & & 0 & \\
\hline 09 & Changguo Rd. No. 27 & & & $\circ$ & & 47 & Sangyuan Aly. No. 11 & & & 0 & \\
\hline 10 & Changguo Rd. No. 29 & & & $\circ$ & & 48 & Changdong No. 9 & & & $\circ$ & \\
\hline 11 & Baihushan Rd. No. 4-6 & & & $\circ$ & & 49 & Changdong Rd. No. 8 & & & 0 & \\
\hline 12 & Xiguanmiao No. 17 & & & $\circ$ & & 50 & Dongguan No. $146-148$ & & & $\circ$ & \\
\hline 13 & Xiguanmiao No. 3 & & & $\circ$ & & 51 & Huanchengnan Rd. No. 99 & & & 0 & \\
\hline 14 & Xiguanmiao No. 16 & & & 0 & & 52 & Renminnan Rd. No. 69,77 & & & 0 & \\
\hline 15 & Changdong No. 1 & & & $\circ$ & & 53 & Yingjuyuan Rd. No. 54 & & & 0 & \\
\hline 16 & Haishi Rd. No. 22 & & & $\circ$ & & 54 & Penglai Rd. No. 111 & & & 0 & \\
\hline 17 & Gujiaqiao No. 11 & & & $\circ$ & & 55 & Yangangxi Rd. 59 & & & 0 & 1 \\
\hline 18 & Haishan Rd. No. 50 & & & . & & 56 & Jinshou No. 4 & & & 0 & \\
\hline 19 & Xiguan No. 5 & & & $\circ$ & & 57 & Changguolu No. 1 to 3 & & & $\circ$ & \\
\hline 20 & Dongdajie No. 131 & $\circ$ & & & & 58 & Changguolu No. 4 to 6 & & & 0 & \\
\hline 21 & Randian Aly. No. 16 & 0 & & & & 59 & Juyuannan No. 28 & & & $\circ$ & \\
\hline 22 & Dongguanmiao No. 36 & & & $\circ$ & & 60 & Juyuannan No. 28 & & & $\circ$ & \\
\hline 23 & Dongdajie No. 119 & 0 & & & & 61 & Weihai Rd. 155 & & & 0 & \\
\hline 24 & Dongdajie No. 135,137 & 0 & & & & 62 & Xiyuan No. 70 & & & $\circ$ & \\
\hline 25 & Dongdajie No. 37 & & & $\circ$ & & 63 & Xiyuan No. 79 & & & 0 & \\
\hline 26 & Furong Aly. No. 5 & & & $\circ$ & & 64 & Dongyuan No. 25 & & & 0 & \\
\hline 27 & Furong Aly. No. 7, 9 & & & $\circ$ & & 65 & Dongyuan No. 18 & & & 0 & \\
\hline 28 & Zhujia Aly. No. 27 & 0 & & & & 66 & Shengjiatang No. 14 & & & $\circ$ & \\
\hline 29 & Chaishui Aly. No. 4 & 0 & & & & 67 & Xiguan No. 11 & & & 0 & \\
\hline 30 & Chaishui Aly. No. 10 & $\circ$ & & & & 68 & Donghai Aly. No. 31 & 0 & & & \\
\hline 31 & Chaishui Aly. No. 13 & $\circ$ & & & & 69 & Hechang Aly. No. 27-3 & & & $\circ$ & \\
\hline 32 & Chaishui Aly. No. 15 & $\circ$ & & & & 70 & Liufang Rd. No. 8 & 0 & & & \\
\hline 33 & Chaishui Aly. No. 22 & 0 & & & & 71 & Dazhong Rd. No. 12 to 13 & 0 & & & \\
\hline 34 & Chaishui Aly. No. 25 & $\circ$ & & & & 72 & Dazhong Rd. No. 10 & 0 & & & \\
\hline 35 & Chaishui Aly. No. 9 & $\circ$ & & & & 73 & Shizhu Aly. No. 33 & $\circ$ & & & \\
\hline 36 & Chaishui Aly. No. 17 & 0 & & & & 74 & Shizhu Aly. No. 9-2 & 0 & & & \\
\hline 37 & Chaishui Aly. No. 27 & $\circ$ & & & & 75 & Shizhu Aly. No. 9-1 & 0 & & & \\
\hline 38 & Chaishui Aly. No. 29 & $\circ$ & & & & & & & & & \\
\hline
\end{tabular}

$\begin{array}{lll}\text { : Commodity housing } & \text { : Public housing involved in DUHR }\end{array}$

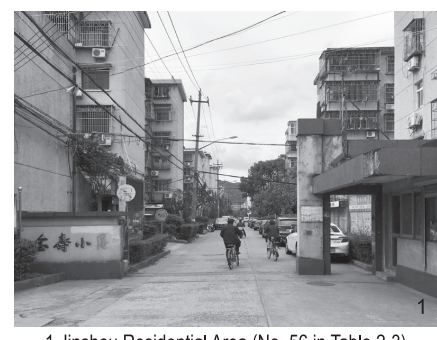

1 Jinshou Residential Area (No. 56 in Table 2-3)

Photo 1, 2 Typical commodity housing and public housing project involved in DUHR in Dinghai Metropolitan Area

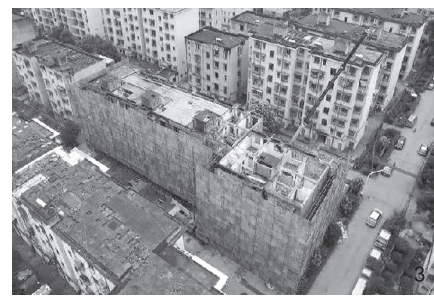

Photo 3 Jinshou No. 4 Building under deconstruction (from Bu-

reau for Housing and Urban-rural Development)

buildings in Jinshou Residential Area have been diagnosed as Class C dilapidated housing ${ }^{* 12)}$ (mapped in Fig. 2).

2) Strategies adopted: Although four strategies ${ }^{{ }^{* 13)}}$ (Table 2-1) are provided by administrative files for homeowners to choose from, overviewing the practice in four cities ${ }^{* 11}$ in Zhejiang Province during 2015 to 2017 (Table 2-2) ${ }^{* 14)}$, most projects have adopted c) and d), while b) has rarely been adopted. ${ }^{* 15)}$ Taking a closer look at the practice in Dinghai Metropolitan Area, strategy a) is only applied to public housing (shaded in grey in Table 2-3), which is not the focus of this paper. Among the private housing projects involved (framed out in Table 2-3), only c) and d) have been adopted. The distinctiveness between private housing projects is only reflected in the proportion of homeowners who accepted c) property swap or d) government buy-back from Table 2-1. Jinshou case (No. 56 in Table 2-3) is first one ${ }^{* 16)}$ of the 75 DUHR projects to be completed in Dinghai metropolitan area in the threeyear DUHR plan according to government files ${ }^{* 8}$. 38 out of 40 homeowners accepted property swap with local government, and 2 homeowners sold their property to the government.

The Jinshou Residential Area is located at the northeast corner of Dinghai metropolitan area. This site is excluded from local government's OCR scheme (Fig. 4, left). Building No.4 was completed in 1987, with brick-masonry structure, 5 storeys, and a total floor area of $2500 \mathrm{~m}^{2}$ (Fig. 3). The building partly collapsed twice in 2012, and residents were evacuated since then. Because there was no practicable mode to cope with Class D Dilapidated Housing, no substantial measures were taken before the DUHR movement carried out in 2015. In 2016, the No.4 building was finally demolished (Photo 3) and transformed into a community parking lot (Photo 4). 


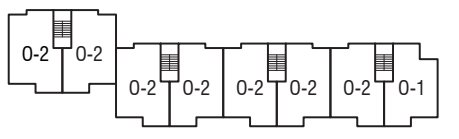

Jinshou No.4 Building

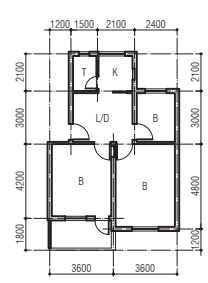

0-1

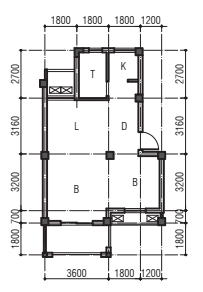

$\mathrm{N}-1$

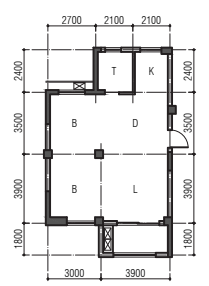

N-6

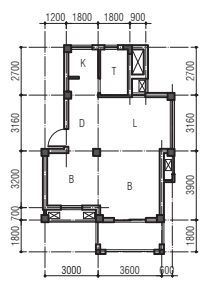

$\mathrm{N}-2$

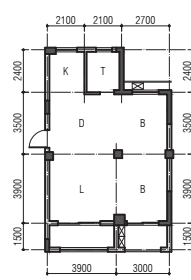

N-7

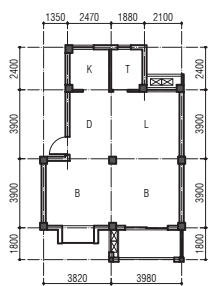

N-3

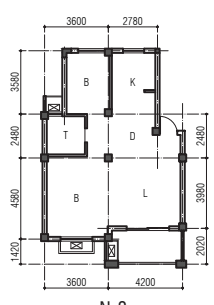

$\mathrm{N}-8$

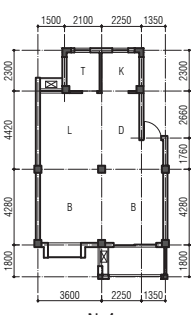

N-4

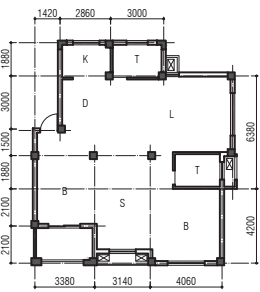

$\mathrm{N}-\mathrm{9}$

Old apartment layouts, Masonry structure Based on measurement of adjacent buildings

New apartment layouts, RC frame structure Collected from Bureau for Urban Planning of Zhoushan

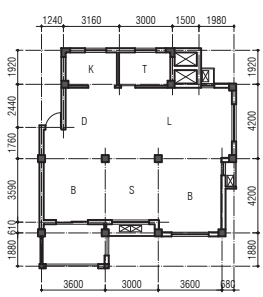

N-5

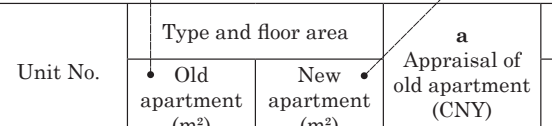

\begin{tabular}{|c|c|}
\hline \multicolumn{2}{|c|}{$\begin{array}{c}\text { b } \\
\text { Price gap paid }\end{array}$} \\
\hline $\begin{array}{c}\text { b1 } \\
\text { by property }\end{array}$ & $\begin{array}{c}\text { b2 } \\
\text { by government }\end{array}$ \\
\hline
\end{tabular}

c
Compensation for
interior finishing and
ancillary facilities of \begin{tabular}{c|c}
$\mathbf{d}$ \\
Reward for \\
contract sign- \\
up (CNY)
\end{tabular} \begin{tabular}{c|c|}
$\mathbf{e}$ & $\mathbf{f}$ \\
Compensation \\
for temporary \\
settlement \\
(CNY)
\end{tabular}$\quad \begin{gathered}\text { Compensation } \\
\text { for relocation } \\
\text { (CNY) }\end{gathered}$ $a+c+d+e+f-$ old apartment (CNY) Part 1. Property swap with local government (38 households)

\begin{tabular}{|c|c|c|c|c|c|c|c|c|c|c|}
\hline A-01 & O-1 (54.72) & $\mathrm{N}-1$ (61.13) & \begin{tabular}{|l|l|}
578,390 \\
\end{tabular} & 158,594 & & 54,419 & 21,888 & \begin{tabular}{|c|}
9,576 \\
\end{tabular} & \multirow{33}{*}{650} & 506,329 \\
\hline A-02 & $\mathrm{O}-2(65.08)$ & $\mathrm{N}-2$ (63.99) & 687,896 & 5,192 & & 75,856 & 26,032 & 11,389 & & 796,631 \\
\hline A-04 & O-2 (65.08) & $\mathrm{N}-2$ (63.99) & 691,150 & & 6,730 & 41,928 & 26,032 & 11,389 & & 777,879 \\
\hline A-05 & O-1 (54.72) & $\mathrm{N}-3(78.41)$ & 583,862 & 385,702 & & 66,715 & 21,888 & 9,576 & & 296,989 \\
\hline A-07 & O-1 (54.72) & $\mathrm{N}-5(100.83)$ & 575,654 & 623,623 & & 41,881 & 21,888 & 9,576 & & 26,026 \\
\hline A-08 & O-2 (65.08) & $\mathrm{N}-5(100.83)$ & 684,642 & 518,274 & & 91,227 & 26,032 & 11,389 & & 295,666 \\
\hline $\begin{array}{l}\mathrm{A}-09 \\
\end{array}$ & O-1 (54.72) & $\mathrm{N}-2$ (63.99) & 567,446 & 138,945 & & 40,010 & 21,888 & 9,576 & & 500,625 \\
\hline A-10 & O-2 (65.08) & $\mathrm{N}-1$ (61.13) & 674,880 & 56,628 & & 53,938 & 26,032 & 11,389 & & 710,261 \\
\hline B-02 & O-2 (64.01) & $\mathrm{N}-1$ (61.13) & 676,586 & & 32,846 & 65,482 & 25,604 & 11,202 & & 812,370 \\
\hline B-03 & O-2 (64.01) & N-3 (78.41) & 679,786 & 229,744 & & 40,296 & 25,604 & 11,202 & & 527,794 \\
\hline B-04 & O-2 (64.01) & N-4 (80.94) & 679,786 & 249,082 & & 67,612 & 25,604 & 11,202 & & 535,772 \\
\hline B-05 & O-2 (64.01) & $\mathrm{N}-3(78.41)$ & 682,987 & 219,315 & & 93,151 & 25,604 & 11,202 & & 594,279 \\
\hline B-06 & O-2 (64.01) & $\mathrm{N}-5$ (100.83) & 682,987 & 483,286 & & 86,111 & 25,604 & 11,202 & & 323,268 \\
\hline B-07 & O-2 (64.01) & $\mathrm{N}-4(80.94)$ & 673,385 & 250,877 & & 54,888 & 25,604 & 11,202 & & 514,852 \\
\hline B-09 & O-2 (64.01) & $\mathrm{N}-3$ (78.41) & 663,784 & 240,348 & & 63,496 & 25,604 & 11,202 & & 524,388 \\
\hline B-10 & O-2 (64.01) & $\mathrm{N}-1$ (61.13) & 663,784 & & 8,264 & 43,211 & 25,604 & 11,202 & & 752,715 \\
\hline $\begin{array}{l}\mathrm{C}-03 \\
\end{array}$ & O-2 (64.01) & $\mathrm{N}-1$ (61.13) & 679,786 & 4,786 & & 77,848 & 25,604 & 11,202 & & 790,304 \\
\hline C-04 & O-2 (64.01) & N-8 (82.17) & 679,786 & 178,730 & & 51,379 & 25,604 & 11,202 & & 589,891 \\
\hline C-05 & $\mathrm{O}-2(64.01)$ & $\mathrm{N}-4(80.94)$ & 682,987 & 219,976 & & 62,188 & 25,604 & 11,202 & & 562,655 \\
\hline C-06 & O-2 (64.01) & N-4 (80.94) & 682,987 & 259,594 & & 63,050 & 25,604 & 11,202 & & 523,899 \\
\hline C-07 & O-2 (64.01) & $\mathrm{N}-4$ (81.02) & 673,385 & 224,121 & & 53,790 & 25,604 & 11,202 & & 540,510 \\
\hline C-08 & O-2 (64.01) & N-9 (122.97) & 673,385 & 713,254 & & 59,011 & 25,604 & 11,202 & & 56,598 \\
\hline $\begin{array}{l}\mathrm{C}-09 \\
\end{array}$ & O-2 (64.01) & $\mathrm{N}-4$ (81.02) & 663,784 & 270,823 & & 70,360 & 25,604 & 11,202 & & 500,777 \\
\hline C-10 & $\mathrm{O}-2(64.01)$ & $\mathrm{N}-3(78.41)$ & 663,784 & 215,776 & & 76,896 & 25,604 & 11,202 & & 562,360 \\
\hline D-01 & O-2 (69.15) & $\mathrm{N}-2$ (63.99) & 730,916 & & 100,171 & 60,518 & 27,660 & 12,101 & & 932,016 \\
\hline D-02 & O-2 (69.57) & $\mathrm{N}-7$ (88.6) & 735,355 & 188,597 & & 56,224 & 27,828 & 12,175 & & 643,635 \\
\hline D-03 & O-2 (69.15) & N-4 (80.94) & 734,373 & 206,502 & & 61,413 & 27,660 & 12,101 & & 629,695 \\
\hline D-04 & O-2 (69.57) & N-3 (78.41) & 738,833 & 149,945 & & 58,943 & 27,828 & 12,175 & & 688,484 \\
\hline D-05 & O-2 (69.15) & $\mathrm{N}-3(78.41)$ & 737,831 & 138,270 & & 43,316 & 27,660 & 12,101 & & 683,288 \\
\hline D-06 & O-2 (69.57) & N-4 (80.94) & 742,312 & 178,525 & & 61,758 & 27,828 & 12,175 & & 666,198 \\
\hline $\begin{array}{l}\mathrm{D}-07 \\
\end{array}$ & O-2 (69.15) & N-3 (78.41) & 727,458 & 264,528 & & 51,956 & 27,660 & 12,101 & & 555,297 \\
\hline D-08 & O-2 (69.57) & $\mathrm{N}-1$ (61.13) & 731,876 & & 89,763 & 80,403 & 27,828 & 12,175 & & 942,695 \\
\hline D-09 & O-2 (69.15) & $\mathrm{N}-1$ (61.13) & 717,086 & & 55,158 & 56,388 & 27,660 & 12,101 & & 869,043 \\
\hline \multicolumn{11}{|c|}{ Part 2. Government buy-back (2 households) } \\
\hline A-03 & O-2 (65.08) & - & 691,150 & - & - & 41,435 & 26,032 & - & \multirow{2}{*}{650} & 759,267 \\
\hline $\mathrm{B}-08$ & $\mathrm{O}-2(64.01)$ & - & 673,385 & - & - & 57,095 & 25,604 & - & & 756,734 \\
\hline Subtotal 2 & & & $1,364,535$ & & & 98,530 & 51,636 & & 1,300 & $1,516,001$ \\
\hline Grand total & - & - & $27,253,067$ & $7,486,233$ & 292,932 & $2,394,028$ & $1,033,264$ & 429,467 & 26,000 & $23,942,525$ \\
\hline
\end{tabular}

Fig. 5 Expenses statement and layouts of apartments in Jinshou Project 


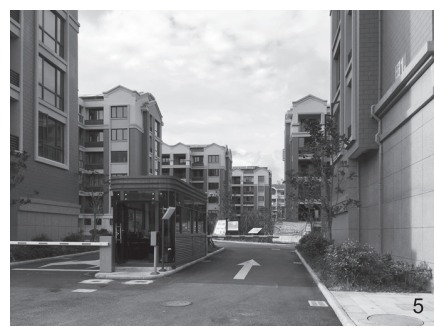

5 Xishanjiayuan

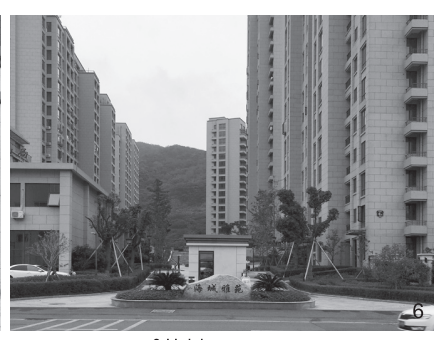

6 Haichengyayuan
Photo 5, 6 Resettlement Housing Areas in Dinghai metropolitan area

$\mathrm{a}=27,253,067$

Trade-in of old apartments $=89.2 \%$

b1-b2-c-d-e-f $=3,310,542$

Price gap paid with money $=10.8 \%$

Fig. 6 Constitution of homeowners' cost for a new apartment in Jinshou Project (calculated based on "Grand total" row in Fig. 5) $\mathrm{a}-(\mathrm{b} 1-\mathrm{b} 2)=20,059,766$

Swapping and purchasing old apartments $=83.8 \%$ $c+d+e+f=3,882,759$ Compensation expenses $=16.2 \%$
Fig. 7 Constitution of government's cost in Jinshou Project (calculated based on "Grand total" row in Fig. 5)

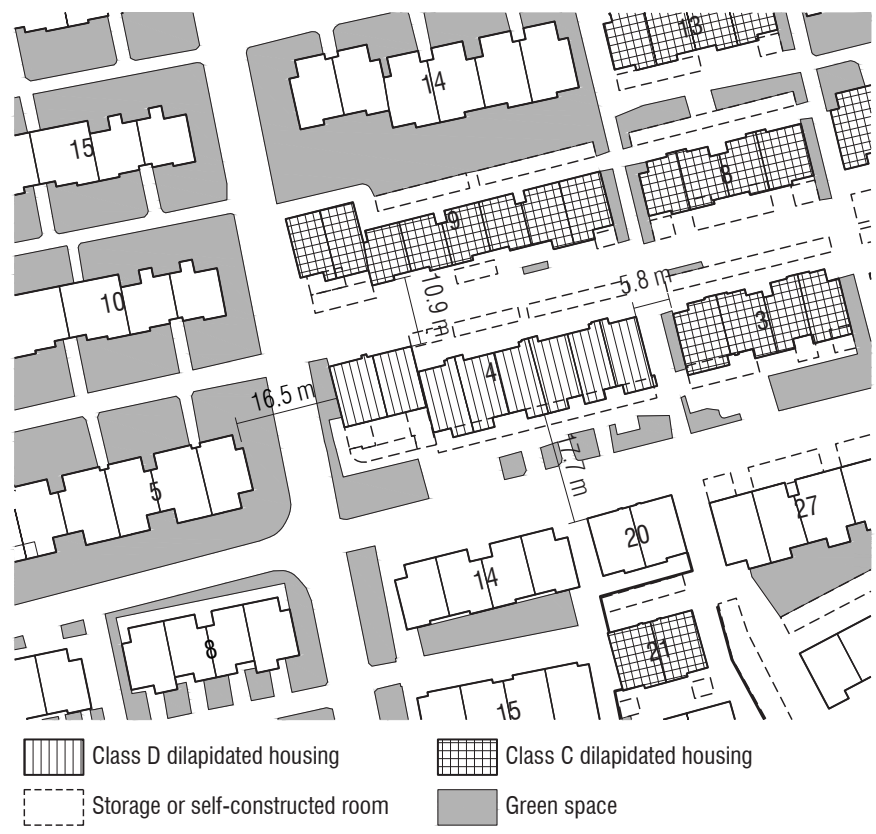

Fig. 8 Distance between Building No. 4 and adjacent buildings

\section{Analysis of financing pattern in Jinshou case study}

In Jinshou Project, for 2 homeowners sold their property to local government, the government acquisition price is based on appraisal of old property (Fig. 5, row A-03 and B-08 in lower table). Though the old building could not be resided any more, government appraisal $10620 \mathrm{CNY} / \mathrm{m}^{2}$ was close to the second-hand house price in nearby areas, which was around $12000 \mathrm{CNY} / \mathrm{m}^{2}$.

For property swap, two residential areas located on the fringe of Dinghai metropolitan area were constructed by local government in advance, specially for relocation households in OCR and DUHR ( $\triangle$ in Fig. 4). New apartment layouts (Fig. 5, upper right) of

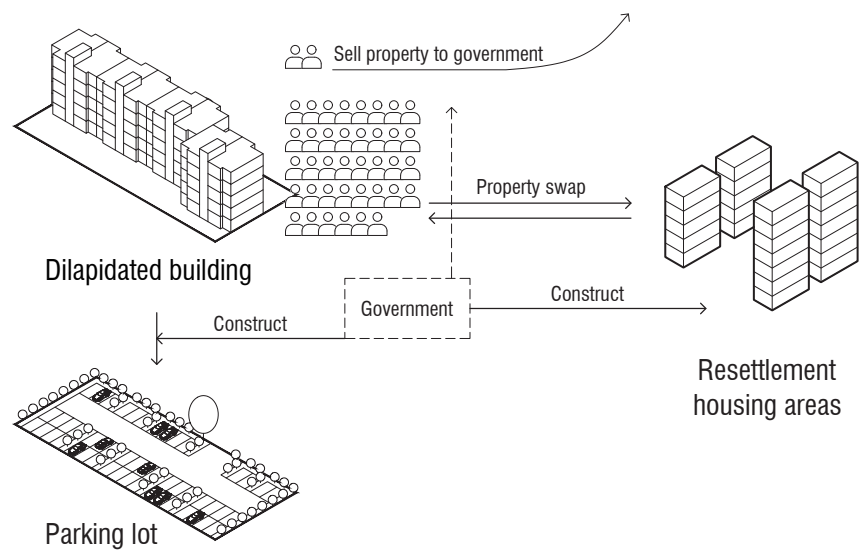

Fig. 9 Summary of financing pattern in Jinshou Project

four standards $\left(60 \mathrm{~m}^{2}, 80 \mathrm{~m}^{2}, 100 \mathrm{~m}^{2}\right.$, and $\left.120 \mathrm{~m}^{2}\right)$ was provided for homeowners to select from. The price gap between old and new apartment was paid in the form of money (Fig. 5, column b). Besides, homeowners received reward for prompt contract signing, as well as compensation for interior finishing and ancillary facilities, temporary settlement, and relocation (Fig. 5, column c, d, e). An equivalent number of 24 million CNY (Fig. 5, last row "Grand total") was spent by local government, in the form of property (new apartments) and money.

From homeowners' perspective, $89.2 \%$ of the cost to get a new apartment was offset by their old apartment and government voluntary compensations, and only $10.8 \%$ were paid in the form of money (calculation shown in Fig. 6). From local government's perspective, $83.8 \%$ of their cost was used to exchange or purchase the old apartments from homeowners, both in the form of new apartment trade-in or money (calculation shown in Fig. 7).

Because the limited government funding is only enough to tackle Class D buildings, other classes could only be monitored regularly by the government at the moment. On the other hand, the distance between building No.4 and the closest building to it is $5.8 \mathrm{~m}$ (Fig. 8). Considering the condition of building foundation of adjacent buildings, it is technically infeasible to construct new apartment building on the site. Consequently, the old building and land that government has acquired is impossible to be redeveloped ${ }^{* 17}$. In this case the old building was transformed into a parking lot (Photo 4) to relieve the parking pressure in Jinshou residential area.

\section{Conclusions and future works}

Through clarifying the financing pattern in Jinshou case study, it could be concluded that government appropriation is the key in current DUHR projects, this mode is isolated from the market and completely relies on government funding. The major part of government expense is used to purchase or exchange the dilapidated properties from homeowners, with a minor part used as subsidy and incitation.

Since the DUHR funding comes from upper-level administrative 
appropriation, local government finance, and bank loans, such financing pattern would lay a heavy burden on different levels of government. With limited fund, only a small part of dilapidated urban housing, i.e. Class D, could be involved, while no essential measure could be taken for other classes. Local government could not retrieve their investment, because the single dilapidated buildings and land they acquired from homeowners could hardly be sold or redeveloped. (Fig. 9) The amount of appropriation is hard to be kept at a steady level in China, so DUHR's capability to meet the increasing need for renewal is in question.

Considering the similarities in terms of strategies adopted in DUHR movement in different areas in Zhejiang Province (as stated in Section 2), many cities-e.g. Shangcheng District, Jianggan District of Hangzhou, Jiangbei District of Ningbo-will encounter the same problem as in Dinghai District of Zhoushan. Besides, the whole country is following the same administration files, while such financing pattern problems will bring severer consequences in regions that are economically less developed than Zhejiang Province. Since the currently diagnosed class D buildings are just a small part of potential buildings that needs to be renewed or reconstructed ${ }^{\left.{ }^{*} 18\right)}$, more sustainable financing patternwith which the government's cost could be retrieved-needs to be explored in further studies.

Local government should not take full responsibility for the problems identified, since the problems to a larger extent originated from the DUHR mechanism, which has led to the dominance of property swap and government buy-back. From the perspective of financing pattern, one of the solutions could be to include surrounding land and buildings in a project, so that the land that local government has acquired could be redeveloped to balance costs. And this solution should be studied together with the marketability of project location, since DUHR projects does not have the best location as in OCR projects. However, current DUHR mechanism does not allow for such solution, so the mechanism needs to be carefully remodeled.

What's more, housing renewal is not merely a matter of economy, the boundary of financing pattern is closely related to the boundary of physical environment improvement, and the DUHR efforts will eventually touch upon physical environment issues not only on a building scale, but also on residential area and urban scale. Therefore in future works, new financing pattern needs to be integrated with new design strategies, including the remodeling of urban structure in old city area, and building codes in China, so that the research findings may really enlighten practical field.

\section{Acknowledgment}

This work is supported in part by scholarship from China Scholarship Council.

\section{References}

1) J. Lue: Beijing's Old and Dilapidated Housing Renewal, Cities, Vol. 14, No. 2, pp. 59-69, 1997

2) J. Lue, P. G. Rowe, and J. Zhang, Eds.: Modern Urban Housing in China, 1840-2000, Prestel, 2001

3) J. Suo: Study on Sustainable Renovation of Urban Existing Housing at Abroad and China, Dalian University of Technology, 2013 (In Chinese)

4) K. Fang and Y. Zhang: Plan and Market Mismatch, Urban Redevelopment in Beijing during a Period of Transition, Asia Pacific Viewpoint, Vol. 44, No. 2, pp. 149-162, 2003

5) M. Shih: The Evolving Law of Disputed Relocation, Constructing Inner-City Renewal Practices in Shanghai, 1990-2005, The evolving law of disputed relocation in Shanghai, International Journal of Urban and Regional Research, Vol. 34, No. 2, pp. 350-364, 2010

6) M. Zhao, Y. Sun, N. Du, and W. Zhao: On the Gradual Urban Renewal, Theory and Practical Strategies, Urban Planning International, Vol. 25, No. 1, pp 24-30, 2010 (In Chinese)

7) X. Luo and J. Shen: Coalition in China's Urbanization, A Case Study of Jiangyin Economic Development Zone in Jinjiang, City Planning Review, Vol. 30, No. 3, pp. 48-52, 2006 (In Chinese)

8) Y. Hu and J. Zhang: Interpretation and Reconstruction of Housing Redevelopment in China-Towards a Just Space Production, China Architecture \& Building Press, 2015 (In Chinese)

9) Z. Qu: The Study on the Renovation of Urban Old Residential Buildings - Take Beijing Qian San Men High-rise Residential Buildings As Example, Tsinghua University, 2011 (In Chinese)

\section{Notes}

*1) Constitution of urban housing stock in China in 1995, 2002, and 2010 (Source: National Bureau of Statistics of China):

\begin{tabular}{l|r|r|r}
\hline Year & \multicolumn{1}{|c|}{1995} & \multicolumn{1}{c|}{2002} & 2010 \\
\hline Proportion of public housing & $56.8 \%$ & $15.6 \%$ & $11 \%$ \\
\hline Proportion of private housing & $40.7 \%$ & $78.5 \%$ & $89 \%$ \\
\hline Others & $2.5 \%$ & $2.5 \%$ & - \\
\hline
\end{tabular}

*2) Chinese private urban housing comes in a variety of forms, this study focuses on the type known as residential areas (“小区”). The dwelling units are outright owned by each homeowner, while the land and ancillary facilities are co-owned by all homeowners. Its form is usually several multi-storey apartment buildings standing in a park-like territory:

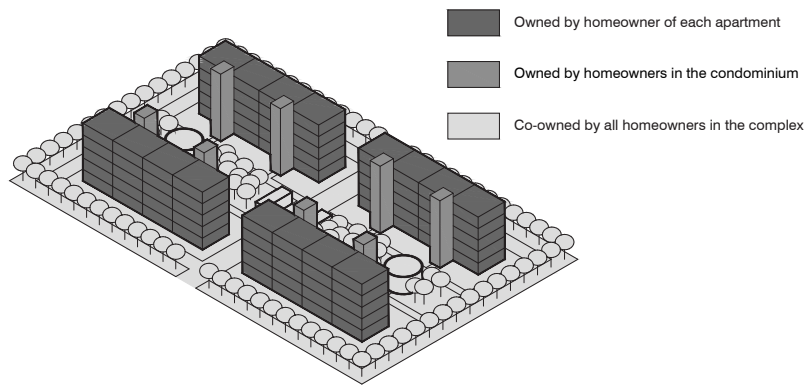

*3) Faced with these issues, this study focuses on housing constructed during early 1980 s to early 1990 s, which in many cities occupies $1 / 4$ of the whole housing stock:

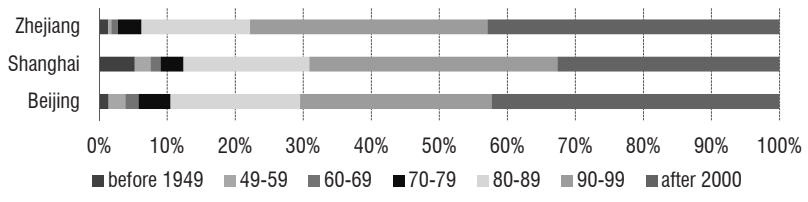

*4) According to Standard of Dangerous Building Appraisal ( 危険 房 屋鑑定標準 JGJ 125-99), dangerous (or dilapidated) buildings (危 房 ) are categorized into four classes (A, B, C, D) according to their physical condition. Class A means the main structure is still safe for daily use though with minor damage; Class B means some structure 
components are in danger, but the whole building is still safe; Class C means a building is partly in danger, and could still be occupied after reinforcement; Class D is the most dilapidated level, indicating the main structure can no longer bear normal use and the whole building is in danger.

*5) On April 4th, 2014, a five-storey condominium constructed in 1994 collapsed in Fenghua, Zhejiang, killing one person while six others were injured.

*6) While there is no uniform term for this mode, it is defined in Urban and Rural Planning Law ( 城 郷 規 劃 法, Article 29 and 31) and Regulation on the Expropriation of Buildings on State-owned Land and Compensation (国有土地上房屋征収和補償条例, Article 8). Old City Renovation (旧城改造) is one of the most commonly used title for this mode.

*7) The FAR limit varies from city to city, location to location. For example, in central area of Hangzhou, the limit could be 4.5, in Shenzhen, the number could be as high as 6.0. Government's planning of FAR limit takes into consideration the profit space for developers, and maximum population density of a region (Liu et al., 2010).

*8) In June of 2015, Chinese State Council carried out Guiding Opinions of the General Office of the State Council on Renovating Dilapidated Housing and Improving Supporting Facilities (国務院関于進一歩做好 城鎮棚戸区和城郷危房改造及配套基礎設施建設有関工作的意見), in order to comprehensively promote the urban renewal process across the nation. The mode of “危房改造” is mentioned in Article 14. A subsidy of 150 billion CNY was appropriated by the central government. A total number of 18 million housing units will be involved from 2015 to 2017.

*9) Different levels of administrative files related to DUHR:

\begin{tabular}{|c|c|c|}
\hline Level & Administrative File & Date \\
\hline Nation & $\begin{array}{l}\text { Guiding Opinions of the General Office of the State Council } \\
\text { on Redevelopment of Shanty Areas and Dilapidated } \\
\text { Buildings and Improving Supporting Facilities } \\
\text { 国務院関于進一歩做好城鎮棚戸区和城郷危房改造及配套基礎 } \\
\text { 設施建設有関工作的意見 }\end{array}$ & 2015.06 \\
\hline Province & $\begin{array}{l}\text { Notice of the People's Government of Zhejiang Province } \\
\text { on Comprehensively Advancing the Work of Renovating } \\
\text { Urban Dilapidated Housing } \\
\text { 浙江省人民政府办公庁関于全面推進城鎮危旧住宅房屋治理改 } \\
\text { 造工作的通知 }\end{array}$ & 2015.06 \\
\hline Municipality & $\begin{array}{l}\text { Implementation Opinions of the People's Government of } \\
\text { Zhoushan on Advancing the Work of Renovating Urban } \\
\text { Dilapidated Housing } \\
\text { 舟山市人民政府関于推進城鎮危旧住宅改造工作的実施意見 }\end{array}$ & 2015.08 \\
\hline District & $\begin{array}{l}\text { Implementation Measures of Dinghai District on } \\
\text { Renovating Urban Dilapidated Housing } \\
\text { 定海区城鎮危旧住宅改造工作実施办法的通知 }\end{array}$ & 2015.11 \\
\hline
\end{tabular}

*10) In China, urban and rural areas follow completely different land and housing systems as shown in the table below. This paper focuses on dilapidated housing issue in urban area.

\begin{tabular}{l|l|l}
\hline & Urban housing (private sector) & \multicolumn{1}{c}{ Rural housing } \\
\hline $\begin{array}{l}\text { Land } \\
\text { ownership }\end{array}$ & $\begin{array}{l}\text { - State owned } \\
\text { rightividuals are granted with use }\end{array}$ & $\begin{array}{l}\text { - Village collectively owned } \\
\text { - Villagers apply to village for } \\
\text { land use right for free (a welfare } \\
\text { system) } \\
\text { - Land use right cannot be } \\
\text { transferred to individuals outside } \\
\text { the village collective }\end{array}$ \\
\hline $\begin{array}{l}\text { House } \\
\text { ownership }\end{array}$ & $\begin{array}{l}\text { - Owned by individuals } \\
\text { - Could be transferred }\end{array}$ & $\begin{array}{l}\text { - Owned by villager } \\
\text { - Cannot be transferred to } \\
\text { individuals outside the village } \\
\text { collective }\end{array}$ \\
\hline
\end{tabular}

*11) Hangzhou is the capital city of Zhejiang Province, with a population of 8700373 (2010). Ningbo is the second largest city in Zhejiang Province, with a population of 7605689 (2010). Shaoxing is a prefecture-level city in Zhejiang Province, with a population of 4912239 (2010). Zhoushan is a prefecture-level city in Zhejiang Province, with a population of 1121261 (2010). The Dinghai metropolitan area covers an area of 12 $\mathrm{km}^{2}$, with a population of 305761 (2010). At present the population of Zhoushan is still growing due to the rapid economic growth in Yangtze Delta Region:

\begin{tabular}{c|r|c|r|r}
\hline Census year & \multicolumn{2}{|c|}{ Total population / growth rate } & \multicolumn{2}{c}{ Total households / growth rate } \\
\hline 1990 & 976132 & - & 302500 & - \\
\hline 2000 & 1001530 & $2.60 \%$ & 360700 & $19.24 \%$ \\
\hline 2010 & 1121261 & $11.95 \%$ & 454800 & $26.09 \%$ \\
\hline
\end{tabular}

*12) By 2017, Building No. 2, 3, 7, 8, 9, 13, 24, 25, 40, 44, 47, 48, 51, 52, $53,54,57,58,68,71,75,76$ are diagnosed as Class C Dilapidated Housing.

*13) The four strategies are specified in administration files listed in Endnote $* 9$. The province level file noted that strategies including structure reinforcement, in-situ reconstruction, government buy-back, etc. could be adopted in DUHR projects. ( ‥三、政策措施：（二）明确 治理改造方式。城镇危旧住宅房屋治理改造应当优先采取以项目形式的成 片改造方式, 也可以采用维修加固、拆除重建或者回购处置等单独改造方 式。…) The municipality level file specified that the renewal of single dilapidated building could adopt structure reinforcement, in-situ reconstruction, property swap, and government buy-back. ( ‥四、主要 任务：… (二) 明确治理改造方式, 分类推进改造工作： …2. 单幢危房改 造根据评估结果采取维修加固、拆除重建、异地置换或者回购处置方式进 行改造。…) The district level file specified the same strategies with the municipality level file. (…第七章 改造方式：…第三十一条…危旧房改造 可以采取维修加固和房屋征收方式。…实施房屋征收的, 可采取原地重建、 异地置换、货币补偿等三种主要方式。…).

*14) The data of Hangzhou, Ningbo, and Shaoxing listed in Table 2-2 for comparing are collected and reorganize from the Office for Dilapidated Housing Renewal of Hangzhou, and Bureau for Housing and Urbanrural Development of Ningbo and Shaoxing, extracted from database of Renewal Strategy for Urban Dilapidated Housing. The data has also been cross referred to aggregate data from Department of Housing and Urban-rural Development of Zhejiang Province. Samples of the original data are shown in the table below:

\begin{tabular}{|c|c|c|c|}
\hline Location & Structure & Strategy & Time \\
\hline$\ldots$ & $\ldots$ & $\ldots$ & $\ldots$ \\
\hline 方山头 26 号 1-4 幢 & 砖混结构 & 异地置换、回购处置 & 2017.05 \\
\hline 卸紫桥9号 & 砖混结构 & 维修加固 & 2017.05 \\
\hline 中学弄 14 号 3 幢 & 砖混结构 & 异地置换、回购处置 & 2017.05 \\
\hline 五里塘路 73 号 & 砖混结构 & 异地置换、回购处置 & 2017.05 \\
\hline 区工商所宿舍 & 砖混结构 & 维修加固 & 2017.05 \\
\hline$\ldots$ & $\ldots$ & & $\ldots$ \\
\hline
\end{tabular}

*15) Nonetheless, there are also exceptions. In Yuecheng District of Shaoxing, the marketized OCR mode has taking a dominant role in redeveloping the old city area. Among the 22 remaining Class D dilapidated housing, 15 of them adopted a), 7 of them adopted c) and d).

*16) The dilapidated housing issue of Jinshou No.4 building had been reported many times in local news before 2015. Therefore local government had a strong will to deal with No.4 building as soon as the DUHR movement is carried out. And this case is implemented as a pilot project to show the virtues of DUHR.

*17) Such close distance between buildings and bad condition of building foundation could be observed in many residential areas of the 1980s.

*18) Take Dinghai Metropolitan Area for example, by 2017, aside from 75 diagnosed Class D Dilapidated Buildings, 465 buildings are diagnosed as Class C Dilapidated Building (Fig. 4), which are also in urgent need of renewal. Besides, the emerging of newly diagnosed dilapidated building is unpredictable. 


\section{和文要約}

1950 年代に確立された中国の福利型都市住宅制度は、1978 年の住 宅改革によって市場主導の住宅制度へと転換された。中国における 現在の都市住宅供給システムでは、民間住宅が主導的な地位を占め ている。本稿では、この民間住宅に関する課題に関して論じる。

急速な都市化の進行に伴う住宅更新に対するニーズの高まりによ り、2015 年以降市場主導の「旧城改造」と政府主導の「危房改造」 による民間都市住宅更新に関する二元的制度が構築された。本稿で は、このシステムにおいて新しい「危房改造」の更新方法に着目し、 資金的側面からこの方法の課題を明らかにすることを目的とする。

本稿の研究対象地域は、浙江省舟山市定海区であり、2015 年から 2017 年までの三年間に実施された「危房改造」による住宅更新を分 析対象とする。また、この住宅更新戦略の特徴から、「金寿新村 4 棟」 を典型的な事例として選定し、物的環境改善を考慮した事業資金統 計の分析を通して、「危房改造」の更新方法の実態を明らかにした。 分析の結果、老朽化した住宅は、地区コミュニティの駐車場へと 転換され、全 40 人の住宅所有者の内 38 人は政府の所有していた不 動産へ移転を承諾し、2人の住宅所有者は地方政府に売却した。地 方政府および住宅所有者の支出と収入の分析により、地方政府は約
2400 万元を一つの更新事業に費やしたことが明らかになった。住宅 所有者の新しい住宅への入居にかかる費用の $89.2 \%$ は、政府の補助 金と住宅売却金により賄われ、10.8\%の費用のみ住宅所有者は自己負 担した。地方政府の $83.8 \%$ の費用は、所有者から住宅を買取あるい は交換するために用いられたが、地方政府により買い取られた土地 は、その小ささから再開発することが難しいと考察される。

本稿では、以下の三点を結論とした。第一に、「危房改造」の更 新方法は、不動産市場からかけ離孔、政府の補助に完全に依存して おり、地方政府の投資は回収できないことが明らかになった。第二 に、「危房改造」による更新方法では、D 級に分類された建物しか適 応されなかったため、住宅地域全体の物的環境の改善に有限的であ る。限定的な政府資金では、老朽化した住宅の一部分しか物的環境 を改善できない。第三に、「危房改造」の更新方法の資金パターンは、 老朽化した住宅数の増加に応じて改善し、政府の負担する費用を回 収できるより持続可能な資金パターンを構築する必要がある。さら に、新しい資金パターンでは、新たな物的環境のデザイン戦略と統 合することが求められるため、本稿の成果は、実践領域において重 要な示唆を与えるであろう。 